\title{
Largest Abdominal CSF Pseudocyst - An Uncommon Complication of VP Shunt
}

\section{Lukhi HD ${ }^{1}$, Singh $M^{1}$, Chakravarthy $S^{1}$, Perwez $N^{2}$}

${ }^{1}$ Department of Radio diagnosis and Imaging, ${ }^{2}$ Department of Neurosurgery, Tata Main Hospital, Bistupur, Jamshedpur, Jharkhand

\begin{abstract}
Abdominal cerebrospinal fluid (CSF) pseudocyst is an unusual and important complication in patients with ventriculoperitoneal (VP) shunt. A 36yrs old male referred to the Department of Radiology for USG abdomen with complaints of gradually increasing distension of abdomen and provisional diagnosis of Alcoholic liver disease. Successive radiological investigations lead to diagnosis of malfunctioning VP shunt, secondary to abdominal CSF pseudocyst formation. Due to lack of suspiciousness patient had developed a giant abdominal CSF pseudocyst, size of which has not been reported in any literature so far. Hence, initial suspicion with appropriate investigation and early treatment can prevent morbidity and mortality.
\end{abstract}

Keywords: Abdominal CSF pseudocyst, Ventriculoperitoneal shunt

\section{Introduction}

Abdominal CSF Pseudocyst formation in patient with VP shunt is an unusual complication and commonly presents with abdominal lump/distension in adult patient and with features of raised intracranial pressure in children. Delay in diagnosis of this complication can lead to progressive increase in size of cyst as it is not lined by epithelium. Hence, no resorption of CSF takes place leading to increased intracystic pressure and malfunctioning of VP shunt

Correspondence to: Dr. Hiteshkumar D. Lukhi, Department of Radio-diagnosis \& Imaging, Tata Main Hospital, Bistupur, Jamshedpur, Jharkhand-831001.

E mail: hitesh.lukhi@gmail.com manifested by hydrocephalus and raised intracranial pressure. Cases have been reported in paediatric age group and very few cases in adults. Lack of early suspicion with background of alcoholic liver disease in

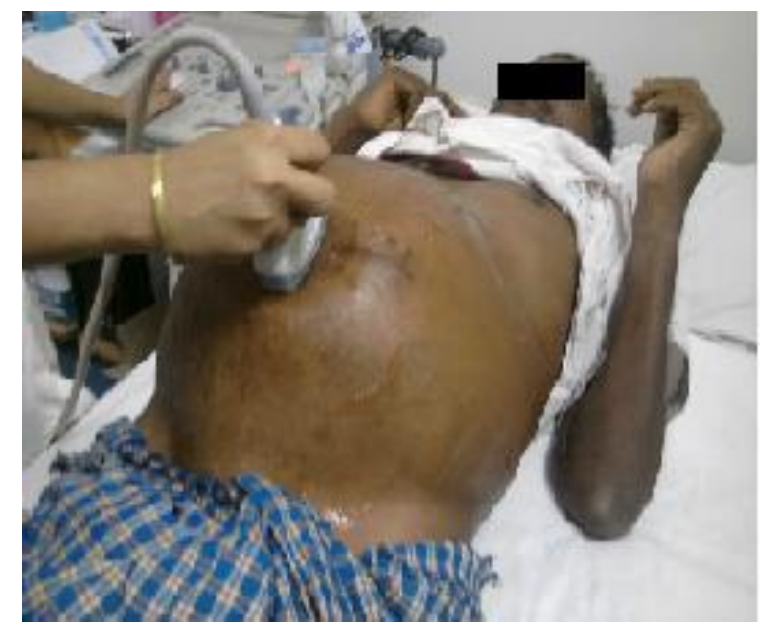

Fig.. 1: Marked distension of abdomen. 


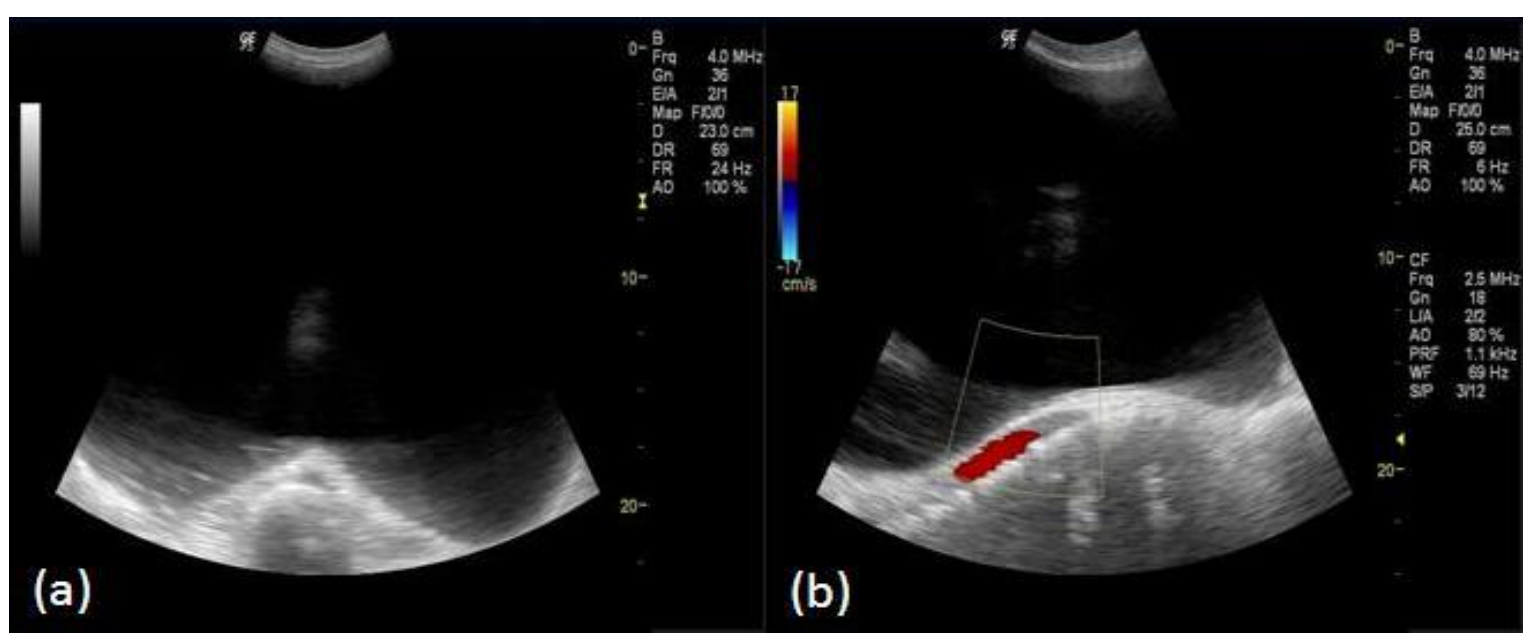

Fig.. 2: Axial (a) and saggital (b) ultrasound images show anechoic fluid filled cyst in the abdomen.

this patient led to delayed diagnosis and formation of abdominal CSF pseudocyst, probably world's largest in size till date.

\section{Case History}

A 36yr old male patient admitted with complaints of gradually increasing abdominal distension and drowsiness since 2 months with no associated fever/gastrointestinal bleeding or past history of any pancreatic pathology/malignancy. Patient was chronic alcoholic since last 20 years with abstinence since last one year. Patient had history of head injury one year back with compound skull vault fracture and brain contusion. Patient underwent craniotomy followed by V-P shunt implantation three months later due to development of post traumatic hydrocephalus. On general examination patient was a febrile, drowsy and vitals were stable with blood pressure of $130 / 90 \mathrm{mmHg}$ and pulse rate of $88 / \mathrm{min}$.

Systemic examination showed no significant cardiovascular or respiratory abnormality. Abdomen was grossly distended (Fig. 1) with presence of fluid thrill and Glasgow
Coma Scale was 13/15 (E4M5V4) at the time of admission. Haematological examination shows normal blood counts with mildly altered liver function test (Alkaline phosphatase-113.3, Alanine aminotransferase-25.2U/L, Aspartate aminotransferase-44.7U/L, Total Serum Protein-7.2 gm/dl, A/G Ratio-0.57, Total Bilirubin-0.52). Thus, general examination and initial lab investigations led to provisional diagnosis of Alcoholic liver disease with ascites and patient was sent for ultrasonography which revealed a large thin walled huge collection (Fig. 2) seen occupying the whole abdomen pushing bowel loops superiorly and kidneys posteriorly with mild fatty infiltration of liver and normal portal vein doppler study. $\mathrm{X}$-ray abdomen showed displacement of bowel loops. Patient underwent CT scan of brain and abdomen which confirmed hydrocephalus and huge abdominal CSF pseudocyst of size $31 \mathrm{~cm} \times 26 \mathrm{~cm} \times 20 \mathrm{~cm}$ (volume 6-7 Litre) with tip of VP shunt in the cyst (Fig. 3).

Patient underwent successful resection of pseudocyst with replacement of VP shunt tip. Patient's post operative period was 


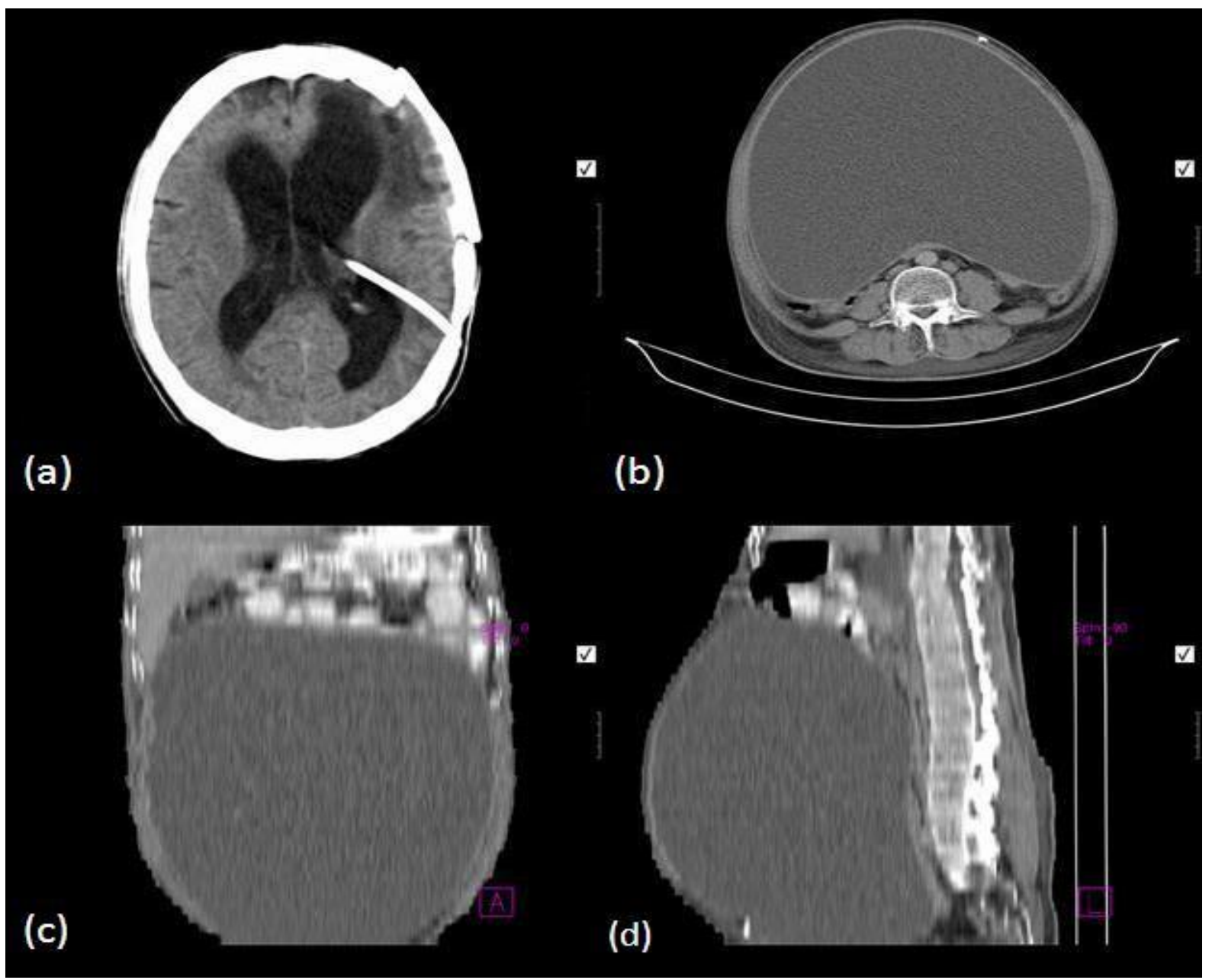

Fig.. 3: CT brain axial scan (a) shows hydrocephalus and VP shunt. CT abdomen axial (b), coronal (c) and sagittal (d) images show giant fluid filled cyst with displacement of bowel loops.

uneventful and was discharged with good general condition. Patient was earlier managed in the line of chronic alcoholic liver disease but imaging changed the diagnosis and management. The abdominal CSF pseudocyst in this patient was perhaps world's largest and size of which has not been reported in literature till date.

\section{Discussion}

The use of the peritoneal cavity for CSF absorption in ventriculoperitoneal shunting was first introduced in 1908 by Kausch. ${ }^{1}$ The most common causes of shunt malfunction are catheter obstruction and infection. The most common distal ventriculoperitoneal shunt complications include shunt infection, recurrent ascites, peritoneal cyst, omental cyst, subphrenic or lesser sac loculation, mesenteric cyst, subcutaneous collection, incisional hernia, intestinal volvulus and intestinal perforation. ${ }^{2}$ Malfunction of the ventriculoperitoneal shunt after initial placement occurs in approximately $25-35 \%$ of patients at 1 year $^{3}$ and $70-80 \%$ of patients require at least one revision at some point in their lives. ${ }^{4}$ Peritoneal CSF pseudocyst formation is an unusual complication with reported incidence of less than $1.0-4.5 \%$. $^{2,5}$ An abdominal CSF pseudocyst was first described by Harsh in $1954 .^{6}$ The wall of the 
pseudocyst is composed of fibrous tissue or an inflamed serosal surface without an epithelial lining and is filled with CSF and debris. $^{2}$ Elevated intracranial pressure and abdominal pain is the most common presentation of an abdominal CSF pseudocyst in children, whereas abdominal pain, lump and, distension predominate in adults. $^{2}$ The time from the last shunting procedure to the development of an abdominal pseudocyst ranges from 3 weeks to 5 years. The CSF pseudocyst can either move freely within the peritoneal cavity or adhere to loops of small bowel, the serosal surface of solid organs or the parietal peritoneum. $^{2}$ We can differentiate CSF pseudocyst from ascites by their characteristic displacement of the bowel gas pattern on abdominal films and by the absence of shifting dullness. ${ }^{2}$ Sonography and CT can accurately localize abdominal fluid collections and other abdominal complication of VP shunt. Fine-needle aspiration of the localized CSF collections under sonographic or CT guidance should be performed to increase the diagnostic yield and though it is not curative, it alleviate the acute symptoms and defer the elective shunt revision. If infection is present, the pseudocyst wall should be excised. ${ }^{2}$ The formation of a CSF pseudocyst is a poor prognostic sign for the usefulness of the peritoneal cavity for shunting. Previous abdominal pseudocyst formation and peritonitis are not contraindications to subsequent peritoneal shunting. ${ }^{7}$ Obstruction of the distal catheter must be treated as an emergency because it can lead to a significant increase in intracranial pressure, resulting in associated complications that can cause considerable morbidity and possibly death. ${ }^{8}$ Nevertheless, knowledge of the broad spectrum of VP shunt complications will enhance the role of radiologists in the management of VP shunt complications. A high degree of suspicion and careful clinicoradiological examination could help diagnose and treat VP shunt complications before they progress to more serious conditions.

\section{References}

1. Kausch W. Die Behandlung des Hydrocephalus der kleinen Kinder. Arch Kiln Chir 1908;87:709-796.

2. Sharma AK, Pandey AK, Diyora BD, Mamidanna R, Sayal PP, Ingale HA. Abdominal CSF pseudocyst in a patient with ventriculo-peritoneal shunt. Indian J Surg 2004;66:360-363.

3. Vinchon M, Fichten A, Delestret I, Dhellemmes P. Shunt revision for asymp-tomatic failure: surgical and clinical results. Neurosurgery 2003;52: 347-353.

4. Blount JP, Campbell JA, Haines SJ. Complicationsin ventricular cerebrospinal fluid shunting. Neurosurg Clin N Am 1993;4:633-656.

5. Oh A, Wildbrett P, Golub R, Yu LM, Goodrich J, Lee T. Laparoscopic repositioning of a ventriculoperitoneal catheter tip for a sterile abdominal cerebros-pinal fluid (CSF) pseudocyst. Surg Endosc 2001; $15: 518$.

6. Harsh GR. Peritoneal shunt for hydrocephalus utilizing the fimbria of the fallopian tube for entrance to the peritoneal cavity. J Neurosurg 1954;11: 284-294. 
7. Latchaw JP Jr, Hahn JF. Intraperitoneal pseudocyst associated with peritoneal shunt. Neurosurgery 1981;8:469-472.

8. Nfonsam V, Chand B, Rosenblatt S, Turner R, Luciano M. Laparoscopic management of distal ventriculoperitoneal shunt complications. Surg Endosc 2008;22:1866-1870. 